\title{
Paralytic poliomyelitis in England and Wales, 1985-91
}

\author{
Rachel Joce, David Wood, David Brown, Norman Begg
}

East and North

Hertfordshire Health

Authority, Welwyn Garden

City, Hertfordshire

AL8 6RD

Rachel Joce, consultant in

communicable disease control

National Institute for

Biological Standards and

Control, Potters Bar,

Hertfordshire EN6 3QG

David Wood, scientist

PHLS Virus Reference

Laboratory, London

NW9 5EQ

David Brown, consultant

microbiologist

PHLS Communicable Disease Surveillance Centre, London NW9 5EQ

Norman Begg, consultant epidemiologist

Correspondence to: Dr Begg.

BMF 1992;305:79-82

\section{Abstract}

Objectives-To ascertain all cases of paralytic poliomyelitis in England and Wales during 1985-91 and to determine the source of infection in each case.

Design-Descriptive study of cases reported between 1985 and 1991.

Setting-All health districts in England and Wales.

Subjects-Patients normally resident in England and Wales whose clinical features were consistent with paralytic poliomyelitis or with laboratory evidence of recent poliovirus infection and compatible symptoms.

Main outcome measures-Clinical, epidemiological, and laboratory features in identified cases.

Results - Of 54 suspected cases of poliomyelitis, 33 were excluded, leaving 21 cases, of which 13 were vaccine associated (nine recipient and four contact) cases, five were imported cases, and three were cases whose source of infection was unknown. No cases due to indigenous wild polioviruses were identified; two were imported cases due to wild viruses. One patient died during the acute phase of the illness, and two children with previously unrecognised severe congenital immune deficiency died between one and two months after the onset of paralysis after the first or second dose of oral polio vaccine. The estimated risk of vaccine associated paralysis is 1.46 per million for the first dose, 0.49 for the second, zero for the third and fourth doses, and 0.33 for the fifth.

Conclusions - Indigenous wild poliovirus seems to have been eradicated, although wild virus may be imported; improved surveillance of suspected cases including immediate notification and characterisation of the virus to ensure that eradication is maintained is essential.

\section{Introduction}

In 1988 the World Health Organisation declared its commitment to the global eradication of poliomyelitis by the year 2000. ${ }^{1}$ In most industrialised nations comprehensive immunisation programmes have succeeded in virtually eliminating the disease. In England and Wales only 70 cases were reported between 1970 and 1984, of which 29 were vaccine associated. ${ }^{2}$ We review the epidemiological, clinical, and laboratory features of cases reported in England and Wales between 1985 and 1991.

\section{Methods}

Ascertainment of cases-Information on suspected cases was obtained by the PHLS Communicable Disease Surveillance Centre from several sources including statutory notifications to the Office of Populations Censuses and Surveys; laboratory reports of poliovirus isolates; and informal reports from consultants in communicable disease control, medical officers for environmental health, microbiologists, and district immunisation coordinators. Detailed information on all suspected cases was sought, including details of immunisation, history of foreign travel, and clinical and laboratory features.

Laboratory investigations-Samples of serum and cerebrospinal fluid were examined for neutralising antibodies to types 1,2 , and 3 poliovirus. ${ }^{3}$ Poliovirus isolates from suspected cases were sent from the source laboratory to the PHLS Virus Reference Laboratory and to the National Institute for Biological Standards and Control for further investigation. The earliest passage of virus was used to characterise strains. Isolates were typed with monospecific polyclonal antisera combined as antiserum pools. At the virus reference laboratory strains were examined by the Van Wezel intratypic serum differentiation method. ${ }^{4}$ At the national institute intratypic differentiation of poliovirus strains was performed with panels of monoclonal antibody. ${ }^{5}$ In one case the strain was also characterised by partial genomic sequencing with the dideoxy primer extension method. ${ }^{6}$

Case definition-Cases included in this review met all three criteria in the following definition. (1) Either the clinical features of the illness were considered to be consistent with paralytic poliomyelitis by the clinician in charge of the case or there was laboratory evidence of recent poliovirus infection in a patient with compatible symptoms. (2) The patient was normally resident in England and Wales regardless of where the infection was acquired. (3) The date of onset of illness was between 1 January 1985 and 31 December 1991.

Epidemiological classification-The WHO classifcation of cases was adopted: vaccine associated, indigenous wild, imported, and source unknown. ${ }^{7}$ Vaccine associated cases were classified as recipient cases if the illness began seven to 30 days after immunisation or as contact cases if the illness began after contact with an individual subject who had been immunised seven to 60 days before the onset of symptoms in the case. ${ }^{8}$

\section{Results}

Fifty four suspected cases of poliomyelitis with dates of onset of the disease between 1985 and 1991 were identified between 1985 and 1991. In 16 of these cases the clinical or laboratory features, or both, were incompatible; in 13 the patients had a non-paralytic illness, and in four non-resident patients had been brought to the United Kingdom specifically for assessment or treatment, or both. These 33 cases were excluded from the review. The remaining 21 cases fulfilled the case definition, 13 of which were vaccine associated cases (nine recipient and four contact), five were imported cases, and three were cases in which the source of the infection was unknown (table I). 
TABLE I-Reported cases of paralytic poliomyelitis, England and Wales, 1985-91

\begin{tabular}{ccccccc}
\hline & \multicolumn{2}{c}{ Vaccine associated } & & & \\
\cline { 2 - 3 } Year & Recipient & Contact & Imported & $\begin{array}{c}\text { Source } \\
\text { unknown }\end{array}$ & Total \\
\hline 1985 & 1 & 0 & 2 & 0 & 3 \\
1986 & 4 & 1 & 2 & 1 & 8 \\
1987 & 0 & 0 & 0 & 1 & 1 \\
1988 & 1 & 1 & 1 & 0 & 3 \\
1989 & 1 & 1 & 0 & 0 & 2 \\
1990 & 2 & 0 & 0 & 0 & 2 \\
1991 & 0 & 1 & 0 & 1 & 2 \\
\hline Total & 9 & 4 & 5 & 3 & 21 \\
\hline
\end{tabular}

\section{VACCINE ASSOCIATED CASES}

Recipient cases-The interval from immunisation to paralysis in the nine recipient cases ranged from 11 to 28 days. Eight of the cases were in infants, of whom six developed paralysis after the first dose of live oral poliomyelitis vaccine and two after the second dose. The ninth case was in a 14 year old boy who developed a footdrop 22 days after a fifth (booster) dose. Viral culture of faeces and serological studies (including testing for cocksackie B $\operatorname{IgM}$ ) gave negative results. Although antibody titres to poliovirus were consistent with previous successful immunisation, the case was included on clinical grounds. At follow up four months after the initial illness he had a typical residual weakness with absent knee and ankle reflexes and normal sensation. Two cases, both fatal, were associated with immune deficiency. One was in a 7 month old boy with severe combined immunodeficiency who died of disseminated poliomyelitis two months after the onset of a flaccid quadriplegia which first appeared 11 days after the second dose of oral poliomyelitis vaccine. Immune deficiency had not been suspected before starting immunisation despite a history of recurrent periorbital and perinasal infections, otitis media, and severe eczema. The second case was in a 3 month old girl with hypogammaglobulinaemia who died from respiratory failure one month after the onset of a flaccid quadriplegia which developed 15 days after the first dose of vaccine. During the early stages of the illness, transient upper motor neurone signs (clonus and increased reflexes and muscle tone) were detected. These features are not typical of polio. Nevertheless, poliomyelitis was recorded on the death certificate, and for this reason the case was included in the review. A postmortem examination was not carried out.

Contact cases-All four contact cases occurred in previously healthy, unimmunised adolescent or adult household contacts of infants who had recently received their first dose of oral poliomyelitis vaccine. The interval from immunisation of the infant to paralysis of the contact ranged from 25 to 52 days.

\section{RISK OF VACCINE ASSOCIATED PARALYSIS}

During 1985-91 about $18 \cdot 4$ million doses of oral poliomyelitis vaccine were administered to children aged 0-15 years in England and Wales. Nine of these recipients developed vaccine associated paralytic polio, a risk of 0.49 per million immunisations (table II). The risk was 1.46 per million for the first dose, 0.49 per million for the second dose, zero for the third dose and fourth (booster) dose, and 0.33 per million for the fifth (booster) dose.

The risk to contacts is more difficult to estimate as the number of contacts per immunised subject is not known. All four contact cases in this study occurred after the first dose. Excretion of poliovirus after the first dose of a primary immunisation series continues for several weeks, and at least one household contact is probably exposed during this time. During the study period $4 \cdot 1$ million children received the first dose of a primary immunisation series. If one contact per immunisation is assumed the risk to contacts was 0.98 per million immunisations for the first dose and zero for subsequent doses. More than one contact may be exposed after each immunisation; thus the true risk of vaccine associated poliomyelitis in contacts may be even lower than these estimates.

IMPORTED CASES

In five cases poliomyelitis was acquired abroad: two cases in Pakistan, two in Bangladesh, and one in Morocco. Four of the cases were in unimmunised subjects, of whom three were children aged between 6 months and 3 years. The fourth case was in a 62 year old previously healthy man who developed quadriplegia and respiratory difficulty eight days after returning from a holiday in a beach resort in Morocco. A wild strain of type 1 poliovirus was recovered from a faecal sample. Three months after the acute illness he remained paraplegic with a permanent tracheostomy. One case was in a 2 year old girl who had previously received four doses of oral poliomyelitis vaccine, the fourth five months before her illness. Serum antibody titres were consistent with successful immunisation; however, she had a typical illness, and a wild strain of type 1 poliovirus was recovered from a faecal sample.

\section{CASES WITH UNKNOWN SOURCE OF INFECTION}

In three cases, there was no history of foreign travel or contact with a recently immunised subject. The first of these was in a 62 year old unimmunised woman who developed severe spinal and bulbar paralysis. No poliovirus was isolated from faeces, urine (two samples each), cerebrospinal fluid or a throat swab (one sample each). Neutralising monotypic type 2 poliovirus antibody was detected in acute and convalescent serum samples (titre 512 in both samples) and in a sample of cerebrospinal fluid (titre 16). The second case was a 30 year old unimmunised man who developed severe spinal and bulbar paralysis. There was no record of examination of appropriate samples for poliovirus; however, neutralising monotypic type 2 poliovirus antibody was shown in acute and convalescent serum samples (titres 128 and 128 respectively). Electromyographic studies were compatible with poliomyelitis, and other conditions which might give rise to the illness such as the Guillain-Barré syndrome were excluded on clinical grounds. The third case was in a 41 year old man who developed a rapidly ascending flaccid paralysis and died within 24 hours of admission to hospital. The cerebrospinal fluid contained $450 \times 10^{3}$ lymphocytes/l (although no poliovirus was isolated) and histological examination of spinal cord and brain tissues at necropsy disclosed severe destruction of cells of the anterior horn. Faecal samples had not been obtained.

\section{LABORATORY INVESTIGATIONS}

Table III summarises the results of laboratory investigations in the 21 cases. Specimens were submitted for viral culture in 18 cases and a poliovirus was isolated in 13 . In 14 cases a faecal sample was

TABLE II -Vaccine associated paralytic poliomyelitis in recipients of oral poliovaccine aged 0-15 years, England and Wales, 1985-91

\begin{tabular}{lccc}
\hline & $\begin{array}{c}\text { Estimated } \\
\text { No of doses } \\
\text { administered } \\
\text { (millions) }\end{array}$ & $\begin{array}{c}\text { No of cases of } \\
\text { paralysis }\end{array}$ & $\begin{array}{c}\text { Risk of paralysis } \\
\text { (per million } \\
\text { immunisations) }\end{array}$ \\
\hline Dose in series & $4 \cdot 1$ & 6 & 1.46 \\
First & $4 \cdot 1$ & 2 & $0 \cdot 49$ \\
Third & $4 \cdot 1$ & 0 & \\
Fourth (booster) & $3 \cdot 1$ & 0 & 0.33 \\
Fifth (booster) & $3 \cdot 0$ & 1 & 0.49 \\
\hline Total & 18.4 & 9 &
\end{tabular}

^Department of Health, unpublished data 
TABLE III - Virological and serological results from 21 cases of paralytic poliomyelitis, England and Wales, 1985-91

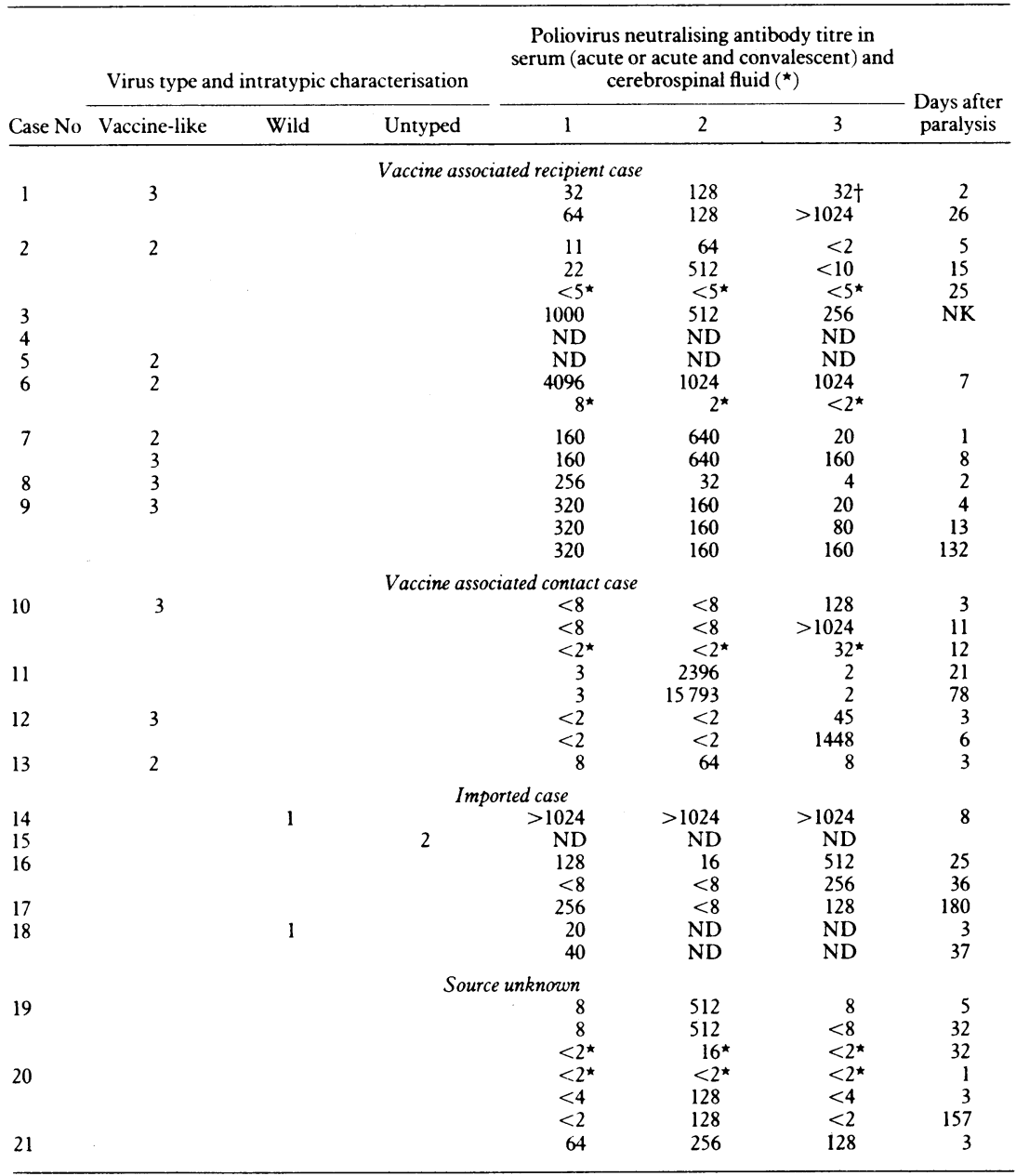

Reciprocal titre. This is a composite table of the results from more than one laboratory.

$\mathrm{ND}=$ not done.

$\mathrm{NK}=$ not known

submitted within two weeks of onset, in $12(86 \%)$ of which poliovirus was isolated. No poliovirus isolates were obtained from urine and throat swabs collected within two weeks of onset from eight cases in subjects positive for the virus.

Only two type 1 viruses were isolated and both were associated with imported cases whereas 10 type 2 or 3 viruses were isolated of which nine were from vaccine associated cases. One type 2 virus was isolated in an imported case, but this patient had been given oral poliomyelitis vaccine after onset of paralysis and before the sample was collected.

Intratypic characterisation of strains from 12 of 13 cases showed that all isolates from vaccine associated cases were vaccine-like whereas the two type 1 viruses isolated in imported cases were wild. Six strains were typed both with monoclonal antibodies and cross absorbed (Van Wezel) sera, and five gave similar results in both tests. In one strain the serological characterisation by Van Wezel sera was confirmed by sequence analysis.

A fourfold or greater rise in antibody titre was shown in paired sera collected from seven cases. In the six cases from which an isolate was obtained the antibody response corresponded to the strain of virus isolated. A significant rise in antibody titre was shown in four of nine recipient cases and three of four contact cases. In the remaining cases only one sample was obtained or the first sample was obtained too late in the illness.

Cerebrospinal fluid was collected and assayed for antibodies to poliovirus in five cases, and in three a low titre of antibody was detected. In one (case 10) there was a raised viral index, suggesting intrathecal production of poliovirus antibody.

\section{Discussion}

The eradication of poliomyelitis requires high levels of vaccine coverage and adequate surveillance to detect every case. Although poliovaccine coverage in England and Wales has now reached $90 \%$ (Department of Health, unpublished data), in some districts coverage is below $70 \%$ and many individuals remain unprotected. It is recommended that no adult should remain unimmunised against poliomyelitis. ${ }^{9}$ No cases of indigenous wild poliomyelitis were identified in this review, although the source of infection could not be found in three cases. Importation of wild virus still occurs, with the potential for transmission among unimmunised subgroups of the population such as itinerants. ${ }^{10}$ Poliovirus isolates from healthy children and from environmental samples should be characterised to guarantee the absence of circulating wild poliovirus. Measures are also required to improve the surveillance of suspected cases. Only nine of the 19 cases in this review were officially notified. Immediate notification of all suspected cases is essential, both to enable timely control measures to be initiated and to maintain adequate surveillance. Since 1991 surveillance of poliomyelitis has been improved by adding acute flaccid paralysis in children to the list of conditions reportable to the British Paediatric Surveillance Unit."

The diagnosis of poliomyelitis should be considered in any patient with a bulbar or flaccid paralysis and appropriate laboratory investigations undertaken. This study confirms the importance of early collection of faecal specimens. As shedding of the virus may be intermittent at least two samples collected $24-48$ hours apart should be obtained as soon as possible after onset of symptoms. Throat swabs should also be taken if the patient is seen within 10 days of the onset of illness, although they did not prove useful in this series. It is important that all isolates from suspected cases should be fully characterised. Serological methods based on monoclonal antibodies and cross absorbed sera are available in the United Kingdom at the PHLS Virus Reference Laboratory and the National Institute for Biological Standards and Control, and the results with both methods show good agreement.

WHO now places less emphasis on detecting neutralising antibodies to poliovirus in suspected cases. ${ }^{5}$ As this study shows, however, this can provide valuable information so we do encourage the collection of acute and convalescent sera. Specimens of cerebrospinal fluid should be obtained from all cases of meningoencephalitis. Although poliovirus is rarely isolated from cerebrospinal fluid, the specimen is necessary for full assessment of the cases. We were unable to establish the value of detecting intrathecally synthesised poliovirus antibodies. ${ }^{12}$ However, a full evaluation of this diagnostic approach is merited, and the potential value of the polymerase chain reaction to diagnosis of poliomyelitis has yet to be assessed. These developments mean that cerebrospinal fluid may become more diagnostically useful in future. Finally, necroscopic specimens (tissues from the brain stem, spinal cord, and descending colon) should be obtained from fatal cases, if possible.

Five imported cases were identified in this series. Although the risk to travellers is small, ${ }^{13}$ poliomyelitis vaccine should be considered for all subjects (irrespective of age) planning to visit countries where transmission of wild poliovirus may still occur. A single booster dose should be given to previously immunised subjects if more than 10 years have elapsed since the last dose.

Two vaccine associated cases were reported in immunodeficient children. Live oral poliomyelitis vaccine is contraindicated for such subjects and their household contacts'; inactivated poliomyelitis vaccine 
should be offered instead. The possibility of immune deficiency should be considered in an infant with recurrent or opportunistic infections.

Despite the very small risk of vaccine associated polio oral poliomyelitis vaccine is one of the safest (and most effective) vaccines in use today. The risk to contacts can be minimised by giving advice to parents of recently immunised babies on the need for strict personal hygiene, particularly washing hands after changing nappies. ${ }^{9}$ Immunising parents (unless previously unimmunised) at the same time as their children is of no additional benefit as the risk of paralysis is similar for vaccine recipients and contacts. Strategies to reduce the risk of the vaccine associated disease even further while retaining the advantages of oral poliomyelitis vaccine are currently being explored - for example, the use of a combined inactivated-live vaccine schedule. ${ }^{14}$ If these strategies are properly implemented the prospects for eradication are good.

1 Global eradication of poliomyelitis by the year 2000. WHO Weekly Epidemiological Record 1988;63:161-2.

2 Begg NT, Chamberlain R, Roebuck M. Paralytic poliomyelitis in England and Wales 1970-84. Epidemiol Infect 1987;99:97-106.
White PMB, Green J. Prevalence of antibody to poliovirus in England and Wales. BMF 1986.293:1153-5.

4 Van Wezel AL, Hazendonk AG. Intratypic serodifferentiation of poliomyelitis strains by strain-specific antisera. Intervirology 1978;11:2-8

5 World Health Organisation. Manual for the virological investigation of poliomyelitis. Geneva: WHO, 1990

6 Evans DMA, Dunn G, Minor PD, Schild GC, Cann AJ, Stanway G, et al. A single nucleotide change in the $5^{\prime}$ non-coding region of the genome of the Sabin type 3 poliovaccine is associated with increased neurovirulence. Nature 1985;314:548-50.

7 Expanded programme on immunisation (EPI): poliomyelitis in 1986, 1987 and 1988. WHO Weekly Epidemiological Record 1989;64:273-80.

8 WHO Consultative Group. The relation between acute persisting spinal paralysis and poliomyelitis-results of a ten-year enquiry. Bull WHO paralysis and polit

9 Department of Health. Immunisation against infectious disease. London: HMSO, 1990.

10 Collingham KE, Pollock TM, Roebuck MO. Paralytic poliomyelitis in England and Wales 1976-77. Lancet 1978;i:976-7.

11 Hall SM, Glickman M. The British Paediatric Surveillance Unit. Arch Dis Child 1988;63:344-6.

12 Hovi T, Stervik M, Kinnuune E. Diagnosis of poliomyelitis by demonstration of intrathecal synthesis of neutralising antibodies. F Infect Dis 1986;153 998-9.

13 Kubli D, Steffen R, Schar M. Importation of poliomyelitis to industrialise nations between 1975 and 1984: evaluation and conclusions for vaccination recommendations. $B M \mathcal{F}$ 1987;295:169-71.

14 National Academy of Sciences Institute of Medicine. An evaluation of poliomyelitis vaccine policy options. Washington, DC: National Academy Press, 1988

\title{
Recruitment methods for screening programmes: trial of a new method within a regional osteoporosis study
}

\author{
M J Garton, D J Torgerson, C Donaldson, I T Russell, D M Reid
}

Department of

Rheumatology, City

Hospital, Aberdeen AB9 8AU

M J Garton, clinical research fellow

D M Reid, consultant

rheumatologist

Health Economics

Research Unit, University

of Aberdeen, Foresterhill,

Aberdeen AB9 2ZD

D J Torgerson, research

fellow

C Donaldson, deputy director

Health Services Research • Unit, University of

Aberdeen, Foresterhill,

Aberdeen AB9 2ZD

I T Russell, director

Correspondence to:

Dr Garton.

BMf 1992;305:82-4

\section{Abstract}

Objective-To estimate the response rates and operating costs of three recruitment methods within a regional osteoporosis screening programme.

Design-Randomised trial of three types of invitation letter: one offering fixed appointments with option to change time, one offering fixed appointments but requiring telephoned confirmation of intention to attend, and one inviting recipient to telephone to make an appointment.

Setting-Osteoporosis screening unit, Aberdeen.

Subjects-1200 women aged 45-49 years living within $32 \mathrm{~km}$ of Aberdeen and randomly selected from the community health index. 400 women were randomised to each appointment method.

Main outcome measures-Numbers attending for screening; default rate among women who confirmed appointments; social class of attenders; cost per appointment slot and per completed scan.

Results-299 (75\%), 277 (69\%), and 217 (54\%) women were scanned after fixed, confirmable, and open invitations respectively. Women who attended were given a questionnaire, and $694(87.5 \%)$ returned it. No significant differences were found in the social class of attenders among the three methods. Of the 514 women who made or confirmed appointments, 494 attended for a scan. Total costs per scan were $£ 25.00, £ 21.40$, and $£ 21.00$ for fixed, confirmable, and open invitations respectively.

Conclusions-The offer of a fixed appointment requiring telephoned confirmation has the potential to reduce the costs of scanning without exaggerating any social bias or significantly reducing response rates provided that empty appointments can be rebooked at short notice.

\section{Introduction}

The NHS operates large scale screening services for carcinoma of the breast and cervix and population screening for osteoporosis is being evaluated in several research centres. To be successful screening requires accurate identification and efficient persuasion of the target population.

Screening by invitation is usually more effective than either opportunistic screening ${ }^{1}$ or self referral, ${ }^{2}$ but which method of invitation best combines good response rates with low cost is uncertain. Only two basic methods of invitation have been reported: fixed appointments with provision to alter the appointment if required and open ended invitations. Government guidelines for breast cancer screening suggest that specific appointments are preferred. ${ }^{3}$ Many studies have assessed the performance of both methods individually, ${ }^{4-11}$ and in the few studies that have compared the two specific invitations consistently outperformed open ended invitations. ${ }^{12-14}$

Response rates to fixed invitations rarely exceed $80 \%$, and so material and human resources are wasted. We compared three different invitation methods for compliance and efficiency within a larger study of population based screening for future osteoporosis by dual energy $x$ ray absorptiometry. At present population screening for osteoporosis remains controversial, and this paper does not address this issue.

\section{Subjects and methods}

The study was approved by the local ethics committee and took place in 1991 at the osteoporosis screening unit, City Hospital, Aberdeen. A single Norland XR26 bone densitometer operated by one full time radiographer was used to scan up to 10 patients a day at the hip and lumbar spine; each scan took about 10 minutes and women were booked in at half hour intervals.

\section{RECRUITMENT}

We identified women aged 45-49 years living in 20 postcode sectors within $32 \mathrm{~km}$ of Aberdeen through the community health index. ${ }^{15} \mathrm{~A}$ proportionate stratified random sample of 1200 women $^{16}$ was randomly 